\title{
Null effect of ginsenoside Rb1 on improving glycemic status in men during a resistance training recovery
}

\author{
Wei-Hsiang Chang ${ }^{1,2,3}$, Ying-Lan Tsai ${ }^{2}$, Chih-Yang Huang ${ }^{4,7}$, City C. Hsieh ${ }^{2}$, Rungchai Chaunchaiyakul ${ }^{5}$, Yu Fang ${ }^{1}$,
} Shin-Da Lee ${ }^{6,8+}$ and Chia-Hua Kuo ${ }^{1,6^{*}+}$

\begin{abstract}
Background: Ginsenoside Rb1, a principle active ingredients of Panax ginseng, has been shown to lower blood glucose in animals and increase insulin secretion in cultured insulinoma cells. The aim of this study was to determine the effects of daily ginsenoside Rb1 supplementation on circulating glucose and insulin levels in men during a 5-day recovery period after an acute bout of resistance exercise.

Methods: Twelve gymnasts ( $20.5 \pm 0.3$ years of age) participated in this double blind placebo-controlled crossover trial. They were challenged by a lower-limb resistance exercise at a weight load of $85 \%$ one-repetition maximal (1-RM) for 10 repetitions, six sets of the movement. Rb1 $(1 \mathrm{ng} / \mathrm{kg})$ or Placebo was orally delivered to participants daily during a 5-day recovery period after challenge. Circulating insulin, glucose and heart rate variability (HRV) were measured under fasted condition in the morning at Days 1, Day 3, and Day 5 during recovery.

Results: No significant effect of Rb1 on circulating glucose and insulin levels were found among participants during the 5-day recovery period. A persistent elevation in sympathetic nervous activity, indicated by increased HRV-low frequency/high frequency (HRV-LF/HF) power, during the Rb1 trial was observed.

Conclusions: The result of the study suggests that the null effect of Rb1 supplementation on lowering glucose and insulin levels of participants may be associated with chronic sympathetic activation.
\end{abstract}

\section{Background}

Existing scientific literatures regarding glucose-lowering effect of ginseng in humans present conflicting results $[1,2]$. Variation in ginsenoside profile associated with different species and cultivating season may account for the discrepancy among previous studies $[1,3]$. Ginsenoside $\mathrm{Rb} 1$ is one of the most abundant ginsenosides in ginseng [4], which has been reported to stimulate insulin secretion in cultured Min6 cells [5] and decrease fasting glucose in rats [6]. To the best of our knowledge, no published data is currently available regarding whether oral $\mathrm{Rb} 1$ supplementation can affect circulating glucose and insulin

\footnotetext{
* Correspondence: kuochiahua@gmail.com

${ }^{\dagger}$ Equal contributors

'Department of Sports Sciences, Laboratory of Exercise Biochemistry,

University of Taipei, Taipei, Taiwan

${ }^{6}$ Department of Rehabilitation Science, China Medical University, Taichung,

Taiwan

Full list of author information is available at the end of the article
}

levels in humans. Pancreatic insulin secretion and insulin sensitivity are, to some extent, modulated by autonomic nervous activity [7-10]. Despite effect of Rb1 on autonomic nervous activity is currently unknown, ginseng extract has been shown to influence the human autonomic nervous system reflected by heart rate variability analysis [11]. It has been reported that exercise containing eccentric contraction elicited a decreased insulin sensitivity in the whole-body glucose disposal [12]. The present work was undertaken to determine the effect of oral $\mathrm{Rb} 1$ supplementation on the glucose and insulin levels in athletes during a 5-day recovery period after a resistance exercise bout containing dynamic (concentric and eccentric) contraction of the lower extremities. Heart rate variability was recorded during a 5-day recovery period.

(C) 2015 Chang et al. Open Access This article is distributed under the terms of the Creative Commons Attribution 4.0 International License (http://creativecommons.org/licenses/by/4.0/), which permits unrestricted use, distribution, and reproduction in any medium, provided you give appropriate credit to the original author(s) and the source, provide a link to the Creative Commons license, and indicate if changes were made. The Creative Commons Public Domain Dedication waiver (http://creativecommons.org/publicdomain/zero/1.0/) applies to the data made available in this article, unless otherwise stated. 


\section{Methods}

\section{Subjects}

Twelve male college gymnasts (aged $20.5 \pm 0.3 \mathrm{y}$; height $169.4 \pm 1.6 \mathrm{~cm}$; weight $63.4 \pm 1.8 \mathrm{~kg}$ ) volunteered to participate in this study. Prior to the recruitment of volunteers, the study protocol was approved by Institutional Review Board of University of Taipei, Taipei, Taiwan. A detailed explanation of the study procedures, including the supplements to be received and potential risks might be involved, was informed to all participants prior to study. All subjects completed a written informed screening questionnaire after explanation. Participants had any form of health problem were precluded. All participants were asked not to change their dietary habit and not to participate in any form of training activity since a week before trial until the end of blood sample collection.

\section{Experimental design}

This study used a placebo-controlled, double-blind, crossover design with a two-week washout period. Participants were randomly assigned to one of two parallel groups, initially in 1:1 ratio, to receive either Rb1 or Placebo under supervision of a lab staff to ensure subject compliance. The Rb1 and Placebo were made by the lab manager in liquid form and were identical in appearance. They were placed in a container and consecutively numbered for each participant according to the randomization schedule. Each participant was assigned an order number and received the drink in the corresponding container. A computer-generated list of random numbers was used for allocation of the participants. A research staff assigned participants to interventions. Except for the interventions, staff was kept blind to supplement assignment of the participants. Staff that takes outcome measurements and staff that delivers the intervention was different. Staffs and participants were maintained masked to outcome measurements and trial results. Before the experiment began, all participants engaged in parallel squatting and their $85 \%$ maximum lower-limb muscle strength (85\% of 1-RM) was assessed. Baseline assessments was conducted under overnight fasted condition. Following a 30-min warm up period consisting of a 5-min warm up, a 10-min full-body stretching, a 5-min lower body stretching, a 5-min low intensity squat practice and a 5-min rest, all participants were challenged with a single bout of high-intensity lower-limb resistance exercise. In brief, this resistance exercise consisted of 6 sets of parallel squats with a resting interval of $60 \mathrm{~s}$ between sets [13]. Each exercise set comprised of a 10-parallel squat repetitions at $85 \%$ one-repetition maximal (1-RM). All participants were instructed to complete each set of squat repetitions within $60 \mathrm{~s}$.

\section{Rb1 supplementation}

Following exercise challenge, each participant was instructed to consume a solution containing ginsenoside Rb1 (or a placebo solution) for 5 consecutive days. Rb1 and Placebo drinks were made available by $0900 \mathrm{am}$ in the morning. Participants orally received the drink once daily in the lab, under supervision of a staff at $0900 \mathrm{am}$ during the 5-day recovery period. The Rb1, provided by NuLiv Science (Walnut, CA, USA), was extracted from Asian ginseng. Rb1 content is confirmed by Prof TsuChung Chang in Department of Biochemistry, National Defense Medical College, Taiwan [14]. Rb1 crystal was solubilized by a $58 \%$ alcohol solution into a concentrate. They were then withdrawn by pipetting into $50 \mathrm{ml}$ of drinking water to achieve a final dose of $1 \mathrm{ng} / \mathrm{kg}$ body weight for each participants. The placebo group consumed same volume of drinking water, which contained an identical amount of alcohol solution. Participants were unable to tell the difference between $\mathrm{Rb} 1$ and Placebo drinks by appearance and taste. The experimental procedure is shown in Fig. 1. The dosage used in this study is equivalent to approximately $0.1 \mathrm{~g}$ of ginseng (wet weight), which is considered low dose.

\section{Samples collection}

Blood samples were collected from fingertip and measured immediately for glucose concentration. Serum sample was used for insulin and cortisol measurements. A total of $200 \mu \mathrm{l}$ of blood was collected from the fingertip for serum preparation, before and 10-min, 1 day, 3 day, and 5 days after resistance exercise challenge [13]. Each blood sample was centrifuged at $4000 \mathrm{rpm}$ for $5 \mathrm{~min}$. Supernatants were used as serum samples for insulin and cortisol measurements. They were frozen at $-80{ }^{\circ} \mathrm{C}$ and analyzed within a week.

\section{Biochemical analysis}

Fasting blood glucose was analyzed using a glucoseoxidase method with the One-Touch glucometer (LifeScan, Milpitas, CA, USA). All hormones were measured using commercially available enzyme-linked immunosorbent assay (ELISA) kits (Diagnostics Systems Laboratories, Inc., Webster, TX, USA) according to the standard procedures provided by the manufacturer. In brief, serum samples (depending on the requirements for specific kits) were loaded into 96-well plates coated with specific antibodies. Once antigens were bound with the specific primary antibody, the secondary antibody was added to form the antibody-antigen-antibody complex. The enzyme was then added and conjugated to the secondary antibody, and the plates were finally developed by adding enzyme substrate to generate visual light signals. The visual light signals were detected by ELISA analyzer (Tecan Genios, Salzburg, Austria). The intra-assay coefficient of 


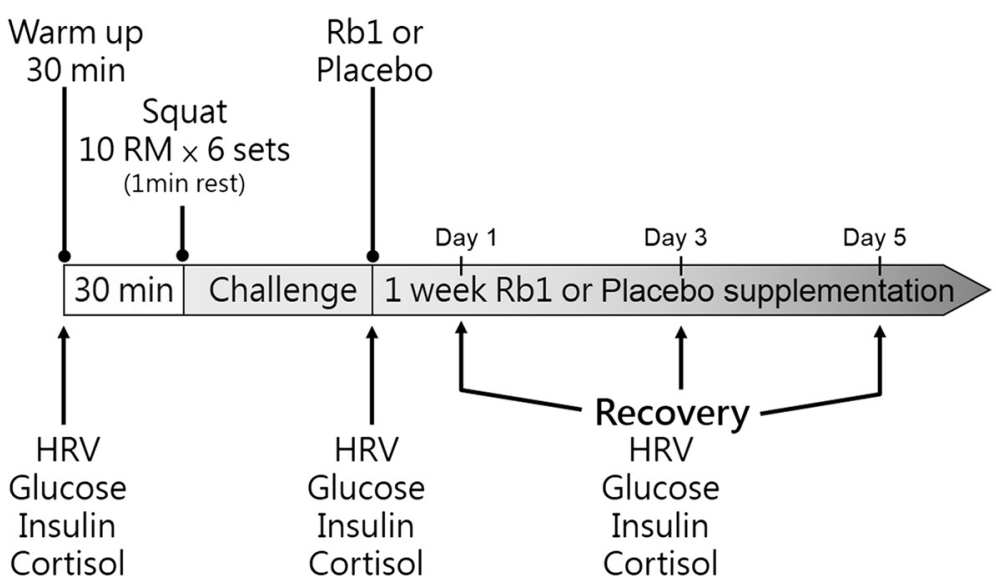

Fig. 1 Experimental procedure. Post-exercise blood sample collection conducted before Rb1 or Placebo supplementation. Pre: before exercise; Post: after exercise; Day 1, Day 3, and Day 5: post-exercise recovery time with Rb1 or Placebo supplementation

variances $(\mathrm{CV})$ for insulin and cortisol were 2.03 and $5.90 \%$, respectively.

\section{Heart rate variability (HRV)}

HRV of all participants was assessed before (09:00), after exercise (11:50) and on the Day 1, Day 3 and Day 5 during recovery period (09:00). HRV was assessed in a quiet, dim environment at room temperature. Prior to assessment, participants were seated and instructed to relax for $5 \mathrm{~min}$. An autonomic nervous system analyzer (Telemedicine Equipment Co., Ltd., Taiwan) was used to acquire a 5-min R-R interval data. HRV-HF was considered to be a measure of parasympathetic nervous system activity, whereas HRV-LF/HF was considered to be a measure of sympathetic nervous system activity [15].

\section{Statistical analyses}

Two-way ANOVA with repeated measure (Rb1 supplementation and time) was performed to assess mean differences in all variables. Fisher's least significant difference test was performed for post hoc comparison. Paired $t$-test was used to compare difference in area under the curve (AUC) of HRV-LF/HF. All values were expressed as mean \pm standard error (SE). The statistical significance was set at $5 \%$ of type I error.

\section{Results and discussion}

Timeline of experimental procedure and blood sampling is illustrated in Fig. 1. Time course data on circulating glucose and insulin are illustrated in Figs. 2 and 3, respectively. Both glucose and insulin concentrations between $\mathrm{Rb} 1$ and Placebo trials were not different throughout the 5 -day recovery period. Cortisol data are illustrated in Fig. 4. No significant difference between trials was found during the 5-day recovery period. Effect of Rb1 on autonomic modulation is based on time course data from
HRV analysis. Rb1 had no significant effect on vagal power (HRV-HF) during the recovery period (Fig. 5). However, sympathetic power (HRV-LF/HF) during Rb1 trial was significantly increased above Placebo level (Fig. 6). A significant trial effect was detected $(P<0.05)$.

We hypothesized that Rb1 would increase insulin and decrease glucose in men after a resistance exercise, based on the existing data showing lowered blood glucose in rats [16] and increased insulin secretion in cultured insulinoma cells [5] after Rb1 treatment. However, we did not detect significant change in circulating glucose and insulin during the 5-day Rb1 human trial. Our result is similar to two studies using crude material of ginseng, which shows no improvement in glucose metabolism of cyclists for a 7 -day $\mathrm{Rb} 1$ trial $[17,18]$. Given the fact that $\mathrm{Rb} 1$ is a major component of ginseng, the result of the study implicates that $\mathrm{Rb} 1$ from ginseng cannot influence

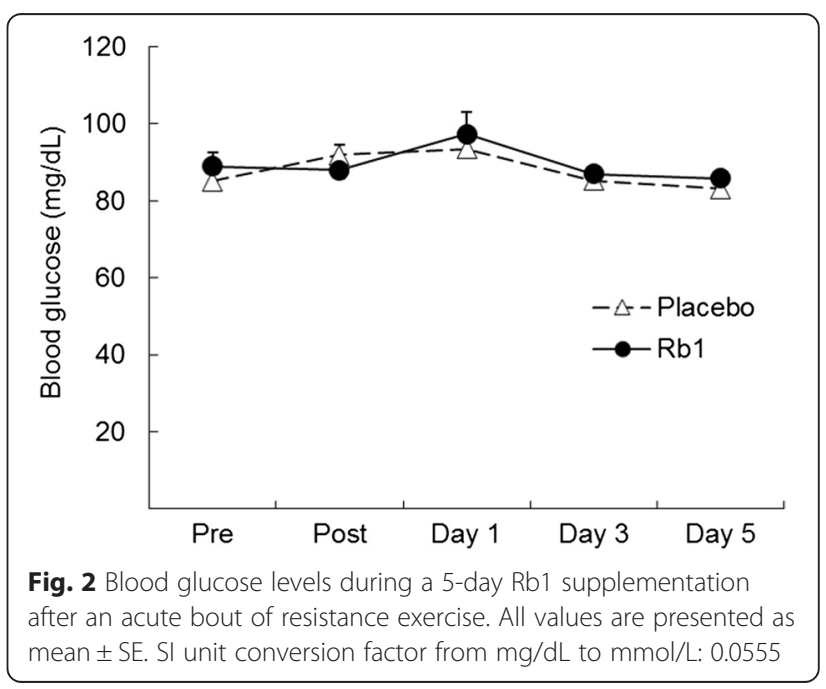




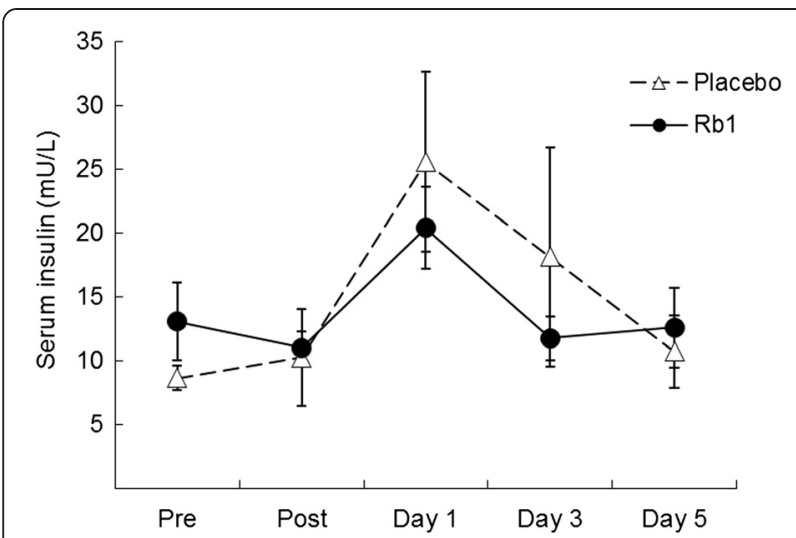

Fig. 3 Serum insulin levels during a 5-day Rb1 supplementation after an acute bout of resistance exercise. All values are presented as mean \pm SE. SI unit conversion factor from $\mu \mathrm{lU} / \mathrm{mL}$ to $\mathrm{pmol} / \mathrm{L}: 6.945$

glucose and insulin in humans during an exercise recovery at this dose.

Sympathetic activation by $\mathrm{Rb} 1$ may be one reason to explain such unanticipated outcome for the absence of glucose-lowering effect. Increased sympathetic activity is generally associated with reduced insulin sensitivity in humans [8], which is also supported by a causal relationship of an increased insulin sensitivity by sympathetic inhibition in obese patients [19]. Ginseng is known as a modulator for the autonomic nervous system [11]. Thus, result of the present study on persistent sympathetic activation suggests that Rb1 is an active component of ginseng and mediated its action via autonomic modulation, which masks our observation on glucose lowering action of Rb1.

Despite ginseng action on glucose metabolism being widely studied subject for human use, most available data come from studies using raw material or extract. One major barrier to establish the knowledge regarding

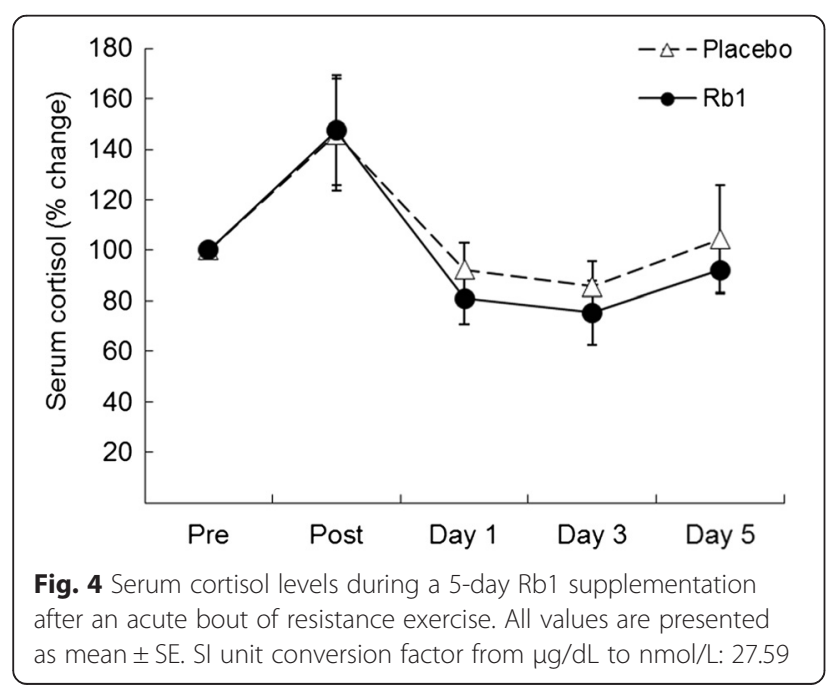

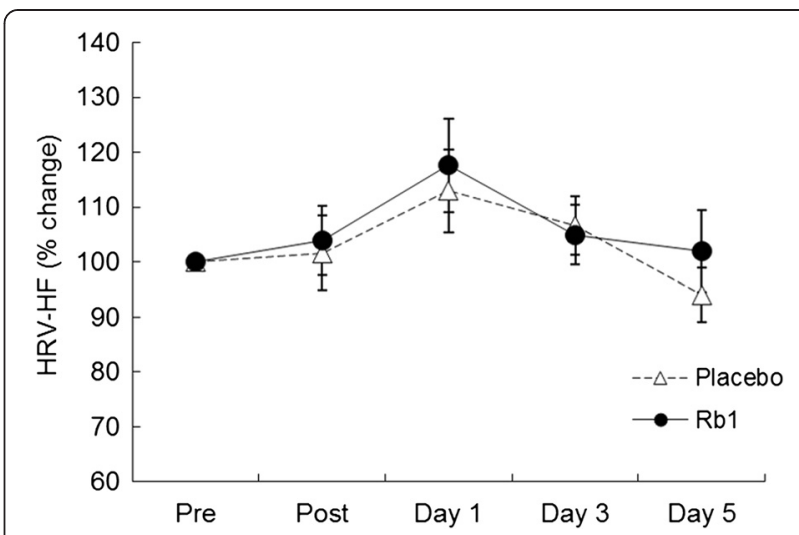

Fig. 5 Vagal modulation (HRV-HF) during a 5-day Rb1 supplementation after an acute bout of resistance exercise. All values are presented as mean $\pm \mathrm{SE}$

whether ginseng can be designed as a hypoglycemic agent is the variation of ginseng source. In a double-blind, randomized, multiple-crossover trial, healthy participants received the same amount of American, Americanwild, Asian, Asian-red, Japanese-rhizome, Vietnamesewild, Sanchi, Siberian ginsengs and Placebo, and showed mixed outcomes on glucose tolerance [1]. Ginseng from the same cultivated land but different batch can also generate inconsistent outcomes on glucose tolerance in humans [3]. Such discrepancy is presumably associated with the changing ginsenoside profile due to season, suggested by the observation that ineffective ginseng batch on improving glucose tolerance also contains decreased ginsenoside content including Rb1 than those effective ginseng batch.

Despite the result of our study does not favor Rb1 as a hypoglycemic component of ginseng as suggested by aforementioned animal studies, we do not preclude the possibility that this ginsenoside component of ginseng may have its claimed action at different doses. The current dose of the study for our human participants was $1 \mathrm{ng} / \mathrm{kg}$. Most of the effective doses tested in previous animal studies are in the range between $5-60 \mathrm{mg} / \mathrm{kg}$, which is substantially greater than what we have been used in the present study $[6,20,21]$. Due to this limitation, dose-response human trial would be needed for further clarification.

Ginseng supplementation has been reported to decrease cortisol levels in humans [22-24]. Since cortisol is a potent stress hormone that stimulates hepatic glucose output and causes peripheral insulin resistance, we were expecting a decrease of cortisol by Rb1 treatment. However, no significant change was detected during the 5-day human trial.

\section{Conclusions}

The present study asks the question whether ginsenoside Rb1 can influence systemic glucose or insulin levels in 


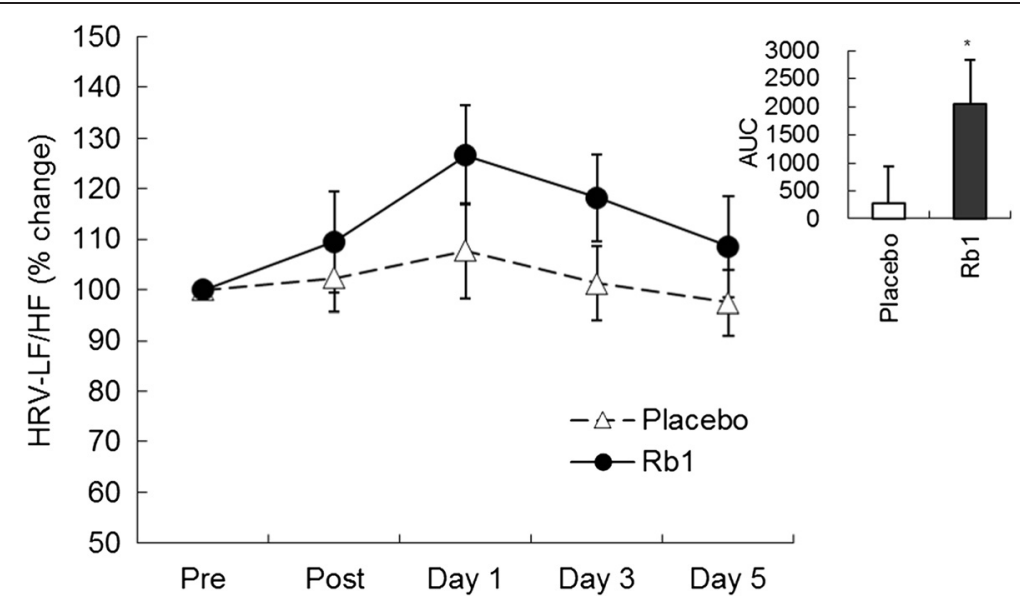

Fig. 6 Sympathetic modulation (HRV-LF/HF) during a 5-day Rb1 supplementation after an acute bout of resistance exercise. AUC: Rb1 $2036 \pm 803$ vs. Placebo $276 \pm 655$. *Significant trial effect $(P<0.05)$. All values are presented as mean \pm SE

humans after an acute bout of resistance exercise, based on the findings of increased insulin release in pancreatic cells and decreased blood glucose level in rats. However, our data did not find any changes in circulating glucose and insulin levels with a low dose Rb1 supplementation. This result may be explained by a persistent elevation in sympathetic nervous activity by Rb1 supplementation.

\section{Competing interests}

The authors declare that they have no competing interests.

\section{Authors' contributions}

WHC, CYH, YF, CCH, YLT, RC, and CHK designed the experiments. WHC and YF performed the experiments. SDL performed the statistical analyses. $\mathrm{CYH}, \mathrm{SDL}, \mathrm{WHC}$ and $\mathrm{CHK}$ wrote the manuscript. All authors read and approved the final manuscript.

\section{Acknowledgments}

We would like to thank Dr. Szu-Hsien Yu for his contributions to the present study. Special thanks to Dr. Yi-Hung Liao for his contributions to our manuscript.

\section{Author details}

'Department of Sports Sciences, Laboratory of Exercise Biochemistry, University of Taipei, Taipei, Taiwan. ${ }^{2}$ Department of Physical Education, National Hsinchu University of Education, Hsinchu, Taiwan. ${ }^{3}$ Department of Athletic Training and Health, National Taiwan Sport University, Taoyuan, Taiwan. ${ }^{4}$ Graduate Institute of Basic Medical Science, China Medical University, Taichung, Taiwan. ${ }^{5}$ College of Sports Science and Technology, Mahidol University, Salaya, Thailand. ${ }^{6}$ Department of Rehabilitation Science, China Medical University, Taichung, Taiwan. ${ }^{7}$ Department of Health and Nutrition Biotechnology, Asia University, Taichung, Taiwan. ${ }^{8}$ Department of Healthcare Administration, Asia University, Taichung, Taiwan.

Received: 7 October 2014 Accepted: 12 August 2015

Published online: 20 August 2015

\section{References}

1. Sievenpiper JL, Arnason JT, Leiter LA, Vuksan V. Decreasing, null and increasing effects of eight popular types of ginseng on acute postprandial glycemic indices in healthy humans: the role of ginsenosides. J Am Coll Nutr. 2004:23(3):248-58.

2. Reay JL, Kennedy DO, Scholey AB. Single doses of panax ginseng (G115) reduce blood glucose levels and improve cognitive performance during sustained mental activity. J Psychopharmacol. 2005;19(4):357-65.
3. Sievenpiper JL, Arnason JT, Leiter LA, Vuksan V. Variable effects of American ginseng: a batch of American ginseng (panax quinquefolius L.) with a depressed ginsenoside profile does not affect postprandial glycemia. Eur J Clin Nutr. 2003:57(2):243-8.

4. Washida D, Kitanaka S. Determination of polyacetylenes and ginsenosides in panax species using high performance liquid chromatography. Chem Pharm Bull (Tokyo). 2003;51(11):1314-7.

5. Park S, Ahn IS, Kwon DY, Ko BS, Jun WK. Ginsenosides Rb1 and Rg1 suppress triglyceride accumulation in 3 T3-L1 adipocytes and enhance beta-cell insulin secretion and viability in min6 cells via PKA-dependent pathways. Biosci Biotechnol Biochem. 2008;72(11):2815-23.

6. Xiong Y, Shen L, Liu K, Tso P, Xiong Y, Wang G, et al. Antiobesity and antihyperglycemic effects of ginsenoside Rb1 in rats. Diabetes. 2010;59(10):2505-12.

7. Carnethon M, Craft L. Autonomic regulation of the association between exercise and diabetes. Exerc Sport Sci Rev. 2008;36(1):12-8.

8. Surwit RS, Feinglos MN. Stress and autonomic nervous system in type 2 diabetes: a hypothesis. Diabetes Care. 1988;11(1):83-5.

9. Porges SW. Vagal tone: a physiologic marker of stress vulnerability. Pediatrics. 1992;90(3):498-504.

10. Jamerson KA, Julius S, Gudbrandsson T, Andersson O, Brant DO. Reflex sympathetic activation induces acute insulin resistance in the human forearm. Hypertension. 1993;21(5):618-23.

11. Yook T, Yu J, Lee H, Song B, Kim L, Roh J, et al. Comparing the effects of distilled rehmannia glutinosa, wild ginseng and astragali radix pharmacopuncture with heart rate variability: a randomized, sham-controlled and double-blind clinical trial. J Acupunct Meridian Stud. 2009;2(3):239-47.

12. Tuominen JA, Ebeling P, Bourey R, Koranyi L, Lamminen A, Rapola J, et al. Postmarathon paradox: insulin resistance in the face of glycogen depletion. Am J Physiol. 1996;270(2 Pt 1):E336-43.

13. Tsai YM, Chou SW, Lin YC, Hou CW, Hung KC, Kung HW, et al. Effect of resistance exercise on dehydroepiandrosterone sulfate concentrations during a 72 -h recovery: relation to glucose tolerance and insulin response. Life Sci. 2006;79(13):1281-6.

14. Chang TC, Huang SF, Yang TC, Chan FN, Lin HC, Chang WL. Effect of ginsenosides on glucose uptake in human caco-2 cells is mediated through altered Na+/glucose cotransporter 1 expression. J Agric Food Chem. 2007;55(5):1993-8.

15. Heart rate variability. standards of measurement, physiological interpretation, and clinical use. Task force of the European society of cardiology and the North American society of pacing and electrophysiology. Eur Heart J. 1996;17(3):354-81.

16. Shang WB, Yu XZ, Wang GQ, Zhao J. Effect of ginsenoside Rb1 in ameliorating insulin resistance and ectopic fat deposition in obese mice induced by high fat diet. Zhongguo Zhong Yao Za Zhi. 2013;38(23):4119-23.

17. Morris A, Jacobs I, McLellan T, Klugerman A, Wang L, Zamecnik J. No ergogenic effect of ginseng ingestion. Int J Sport Nutr. 1996;6(3):263-71. 
18. Reeds DN, Patterson BW, Okunade A, Holloszy JO, Polonsky KS, Klein S. Ginseng and ginsenoside Re do not improve $\beta$-cell function or insulin sensitivity in overweight and obese subjects with impaired glucose tolerance or diabetes. Diabetes Care. 2011;34(5):1071-6.

19. Gamboa A, Okamoto LE, Arnold AC, Figueroa RA, Diedrich A, Raj SR, et al. Autonomic blockade improves insulin sensitivity in obese subjects. Hypertension. 2014;64:867-74.

20. Tan SJ, Li N, Zhou F, Dong QT, Zhang XD, Chen BC, et al. Ginsenoside Rb1 improves energy metabolism in the skeletal muscle of an animal model of postoperative fatigue syndrome. J Surg Res. 2014;191(2):344-9.

21. Yang CY, Wang J, Zhao Y, Shen L, Jiang X, Xie ZG, et al. Anti-diabetic effects of panax notoginseng saponins and its major anti-hyperglycemic components. J Ethnopharmacol. 2010;130(2):231-6.

22. Popov I, Goldwag W. A review of the properties and clinical effects of ginseng. Am J Chin Med (Gard City NY). 1973;1(2):263-70.

23. Bahrke M, Morgan W. Evaluation of the ergogenic properties of ginseng. Sports Med. 1994;18(4):229-48.

24. Odani T, Ushio Y, Arichi S. The effect of ginsenosides on adrenocorticotropin secretion in primary culture of rat pituitary cells. Planta Med. 1986;3:177-9.

\section{Submit your next manuscript to BioMed Central and take full advantage of:}

- Convenient online submission

- Thorough peer review

- No space constraints or color figure charges

- Immediate publication on acceptance

- Inclusion in PubMed, CAS, Scopus and Google Scholar

- Research which is freely available for redistribution 